\title{
Low Velocity Impact Response on GFRP and GF-SMP Panels in Structural and High Frequency Bands
}

\author{
R. Basavanna' ${ }^{1}$, S. Raja ${ }^{2 *}$, Karisiddappa ${ }^{3}$ \\ ${ }^{1}$ Civil Engineering Department, Visvesvaraya Technological University, Belagavi, India \\ ${ }^{2}$ Structural Technological Division, CSIR-National Aerospace Laboratories, Bangalore, India \\ ${ }^{3}$ Visvesvaraya Technological University, Belagavi, India \\ Email: ^raja@nal.res.in
}

How to cite this paper: Basavanna, R., Raja, S. and Karisiddappa (2020) Low Velocity Impact Response on GFRP and GF-SMP Panels in Structural and High Frequency Bands. Advances in Aerospace Science and Technology, 5, 58-70. https://doi.org/10.4236/aast.2020.52004

Received: April 23, 2020

Accepted: June 25, 2020

Published: June 28, 2020

Copyright $\odot 2020$ by author(s) and Scientific Research Publishing Inc. This work is licensed under the Creative Commons Attribution International License (CC BY 4.0).

http://creativecommons.org/licenses/by/4.0/

\begin{abstract}
A low velocity impact response study has been carried out on glass fibre composite laminates, made up of regular aircraft grade epoxy (GFRP) and shape memory polymer (GF-SMP). Under various impact loading intensities (4J, 6J, 12J), the responses are measured by a network of PZT (Lead Zirconate Titanate) sensors. A signal analysis methodology is subsequently developed to process the very high frequency $(60 \mathrm{MHz})$ sampled data. In two frequency bands, namely $0-2000 \mathrm{~Hz}$ and $0-100 \mathrm{KHz}$, the results are examined and the transient dynamic behaviours of the composite laminates are evaluated. It is observed that both the laminates have generated the high frequency structural waves $(0-100 \mathrm{KHz})$, which can be exploited to examine the BVID. However, GF-SMP laminate has shown some advantage in terms of energy dissipation in the structural frequency band $(<2000 \mathrm{~Hz})$. Further, the GF-SMP laminate has demonstrated its capability to generate very high frequency structural waves, which could carry the damage information like BVID due to impact event to nearby sensors for impact event monitoring and health assessment.
\end{abstract}

\section{Keywords}

Impact, Low Velocity, PZT Patches, GFRP, Composite Laminate, Transient Dynamics

\section{Introduction}

Fibre Reinforced Composite Structures may undergo various service loads during the operational time of the aircraft, space and automotive vehicles. These dynamic loads over a period of time may cause different kinds of damages in the 
structures, namely matrix crack, fiber pull-out, delamination, de-bonding of stiffeners and fatigue. Besides the operational (maneuvering) loads, accidentally the aerospace structures can also experience impact events; for example, birds' strike. Therefore, it is mandatory to qualify these aircraft structures for impact loads, according to the Federal Aviation Regulations (FAR). It really demands the application of both numerical and experimental methods to be applied during the design phase of the structures, to ensure the safety margin. Conventional Non-destructive methods (C-Scan, Acoustic Emission, Thermography, Eddy Current Probe, Vibration Analysis etc.) are being used by aerospace industries to qualify the manufactured components, as well as they are deployed in periodical inspection for maintenance.

Researchers worldwide continuously update their understanding in mechanics of composite materials and structures, as the synthesis of new materials and modern manufacturing techniques are fast emerging [1]. A review article is presented by Richardson and Wisheart [2] on low velocity impact properties of composite materials, focusing on major impact induced damage modes. They have further highlighted the effects of the composite constituents on impact properties, assessing the post impact performance through residual strength. Schuchang et al. [3] have predicted the delamination in composite laminates under low velocity impact using hybrid approach. FEM based damage model is validated with the results of drop weight tests and ultrasonic C-scan images. The major effort is put to visualise the shape of the delamination by cohesive contact method. There are notable research articles reported in the area of impact damage identifications [4]-[9]. Tetra hertz waves are used to build images by a quantum cascade laser to detect delamination and crack in the fiber fabrics [10]. The results are compared with conventional C-Scan NDT to show the merits of the developed approach.

A paradigm shift has taken place in the last two decades due to a new diagnostic approach known as "Structural Health Monitoring (SHM)", in which discrete/distributed sensors are employed to collect the real time structural responses, namely displacement, strain, pressure, acoustic emission and very high frequency response etc. SHM is currently becoming a promising area, where the health status of a structure is monitored through an array of sensors and subsequently the collected data is periodically diagnosed for identifying the damage, if present. Different damage detection and monitoring methodologies are evolved using mechanical/electrical/acoustical information, adopting statistical method, signal processing technique and probabilistic approaches etc. SHM has given a new thrust to the design of aircraft structures and mechanical systems by reducing their maintenance cost and improving safe operation during the service [11].

The occurrence of damage in aircraft structure or mechanical system may change its material or geometric properties, which may severely affect the performance of the system. Therefore, a periodical assessment of the system states in time scale is very much essential, in order to evaluate the influence of damage on the health of the system [12] [13]. SHM adopts a different kind of sensors and 
piezoelectric materials are the prominent ones among them. The Lamb wave propagation can be initiated and the same is received by using piezoelectric emitters and sensors, respectively. The Lamb wave with a specified high frequency (wave length) can travel in thin plates that may capture the damage information along its travelling path/media. Thus, Lamb wave can act as a carrier of the required damage information like BVID in the structure for diagnostic purpose. There are two fundamental Lamb wave modes (A0 and S0) existing, which can be effectively generated through thin piezoelectric patches as emitters. Ajay and Cesnik [14] and Su et al. [15] have presented a detailed survey of different works carried out on the application of guided Lamb waves in SHM. In a recent study, the CNT nanomaterial based omni-directional sensor technology is developed to detect an impact related damage in a wider area of composite structures [16].

Epoxy, a thermoset polymer of different grades is generally used for making aerospace composite structures. To improve the impact resistance of composites, many approaches are proposed; namely, introducing nano-materials into polymers, use of viscoelastic layer/patches, design of metallic leading edges etc. In recent years, shape memory polymers (SMP) are emerging as the functional polymeric systems and they have already been found applications in bio-medical and other engineering disciplines [17] [18]. Indeed, SMP may find suitability in aerospace composite structural application in future due to its multifunctional properties, such as shape memory/recovery, higher flexibility and material damping [19] [20]. Thus, SMP with proper glass transition temperature can be considered as a functional resin system in an advanced composite structural formation.

The current research is focussed on fabrication of glass fiber reinforced laminate using multifunctional SMP matrix system (GF-SMP) and a comparison study is made with a conventional GFRP laminate, which is made of LY556. The dynamic characteristics are established through vibration testing for both the cases to show the applicability of SMP resin in laminated structures. Subsequently, the performance of GF-SMP laminate is examined under low velocity impact loading. The impact responses are critically assessed in two frequency bands, namely low frequency ( 20 to $2000 \mathrm{~Hz}$ ) to cover the responses of structural modes and to investigate the high frequency lamb waves due to impact event (10 to $100 \mathrm{KHz}$ ). The focus of this research has been laid on the development of multifunctional laminate using a functional resin system with glass fiber fabric. So that the laminated structure will have an in-built energy dissipation capability and self-informing nature through a piezoelectric sensory network. For that reason, the transient dynamic nature is evaluated in both low frequency and very high lamb wave frequency bandwidths under impact loadings.

\section{Fabrication of GF-SMP and GFRP Composite Panels}

To conduct the low velocity impact experiments, composite laminates $(200 \times$ $\left.200 \times 1.5 \mathrm{~mm}^{3}\right)$ are fabricated using GFRP bi-directional fabric (7 mil) with a 
conventional thermoset epoxy (LY556) and an in-house developed shape memory polymer (SMP) [19]. An aluminium plate of thickness $(3 \mathrm{~mm})$ is used as a supporting structure for the composite laminate, on which it is adhesively bonded as shown in Figure 1 and Figure 2(a), Figure 2(b), respectively. GF-SMP laminate is cured for 10 minutes under UV light first and then post cured in an oven at $90^{\circ} \mathrm{C}$ temperature for $1 \mathrm{hr}$.

The test specimens are instrumented with four piezoelectric patches $(10 \times 10$ $\times 0.5 \mathrm{~mm}^{3}$ ) to collect the impact induced time responses. Additionally, one accelerometer (sensitivity $10 \mathrm{mV} / \mathrm{g}$ ) and a strain gauge are bonded to measure the intensity of the impact events.

\section{Experiments}

Two types of experiments are conducted on the composite laminates, namely 1) Vibration testing, and 2) Impact testing. Through vibration measurements, the stiffness is compared for both GFRP and GF-SMP composite laminates. On the other hand, impact responses are expected to show the role of flexible resin system and its significance in the mechanics of composites. For that purpose, the digital signal processing technique is used to clearly distinguish the behaviour of both composites under transient loading, in two different frequency bands (low frequency in $\mathrm{Hz}$, high frequency in $\mathrm{KHz}$ ). Also, it is worth noticing that the developed DSP based approach will provide the necessary route to extract the high frequency information, which is capable of giving the occurrence of transient events and its effect properly.

\subsection{Vibration Testing}

Impulse test is conducted on the composite specimens using a hammer ( $\mathrm{PCB}$ $086 \mathrm{C} 1$ ) with a force transducer (sensitivity $11.2 \mathrm{mV} / \mathrm{N}$ ). The response is collected in the form of frequency response function (FRF). For this purpose, the commercially available Siemens-LMS ${ }^{\varpi}$ multi-channel data acquisition system is used and the collected data is processed through modal analysis in Test. Lab software to estimate the natural frequencies. The plates are mounted on the fixture as shown in Figure 3(b). The vibration test results are used to examine the dynamic similarity of the two laminates, which are made by conventional epoxy and shape memory polymer (SMP).

From Table 1, it can be seen that both the laminates appear to be dynamically similar, except the first mode frequency, showing a moderate deviation. It is therefore evident that SMP resin does not drastically reduce the stiffness property of the laminate.

\subsection{Impact Testing}

The impact response study is conducted using a low velocity impact test facility, as shown in Figure 3(a). It has got the vertical pillars that hold the impactor mass at a required height. The height is calibrated to have different impact intensities, 
namely 4, 6, 9, and 12 Joules, respectively. The setup has a release mechanism for making the required impact on the plate specimens. Both the laminates are subjected to similar impact intensities at the same locations, so that a comparison can be made later to evaluate the benefits of flexible SMP over conventional epoxy. The focus here is to assess the impact response behaviour of the laminates both in structurally dominant low frequency segment and also in the high frequency lamb wave band. Figure 3 explains the facility, instrumented laminate, and various impacted locations.

Table 1. Comparison of natural frequencies.

\begin{tabular}{cccc}
\hline \multirow{2}{*}{ Sl.no. } & Frequency $(\mathrm{Hz})$ & Frequency $(\mathrm{Hz})$ & \\
\cline { 2 - 3 } & GF-SMP $(F)$ & GFRP $(f)$ & $\frac{F-f}{F} \times 100$ \\
\hline 1 & 175.0 & 160.5 & $8.28 \%$ \\
2 & 270.0 & 270.5 & $0.18 \%$ \\
3 & 308.5 & 318.0 & $2.98 \%$ \\
4 & 547.0 & 545.0 & $0.37 \%$ \\
5 & 693.0 & 691.5 & $0.22 \%$ \\
6 & 794.0 & 793.0 & $0.13 \%$ \\
\hline
\end{tabular}
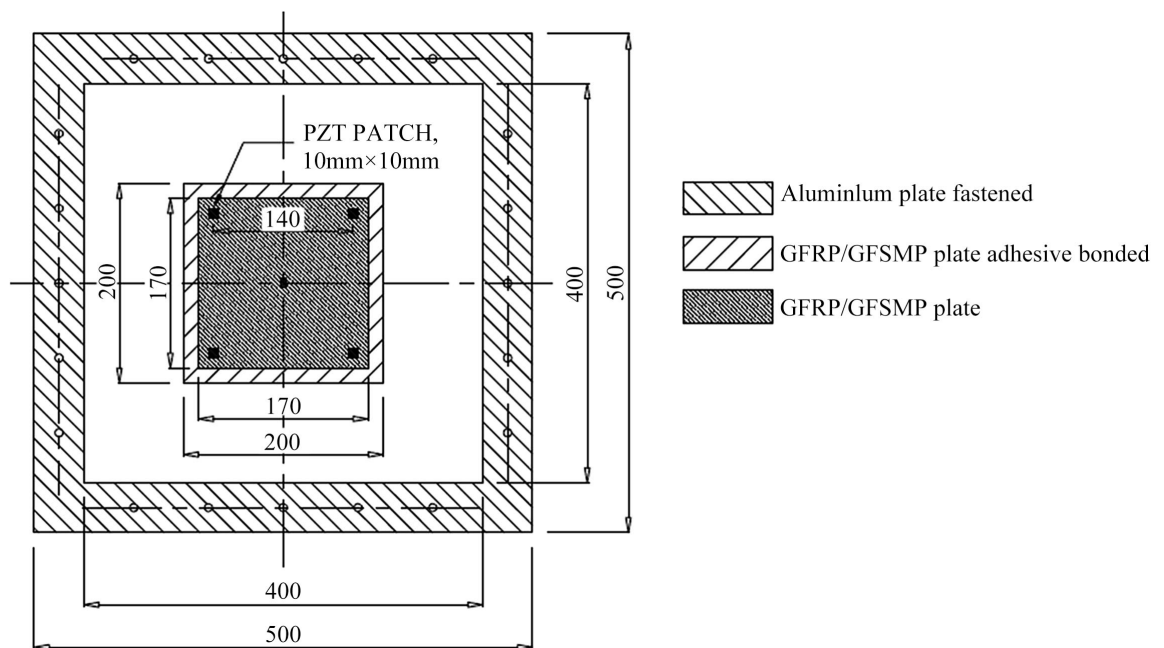

Figure 1. Specimen details.

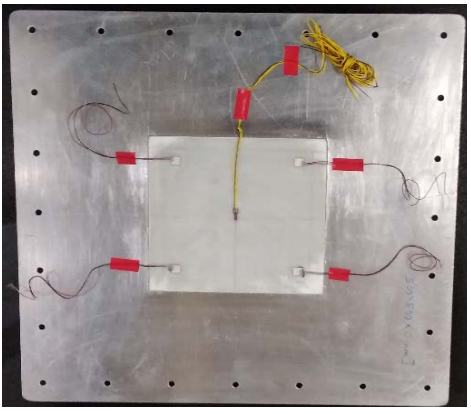

(a) GF-SMP panel

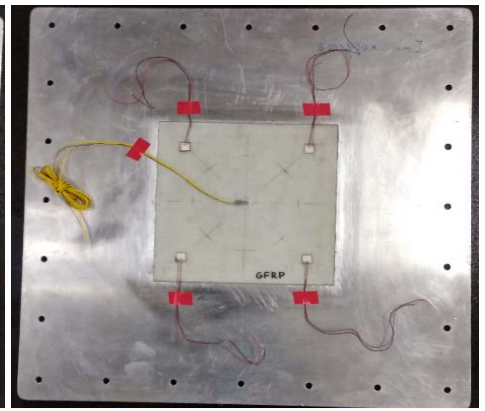

(b) GFRP panel

Figure 2. Fabricated composite panels, bonded on aluminium fixture. 


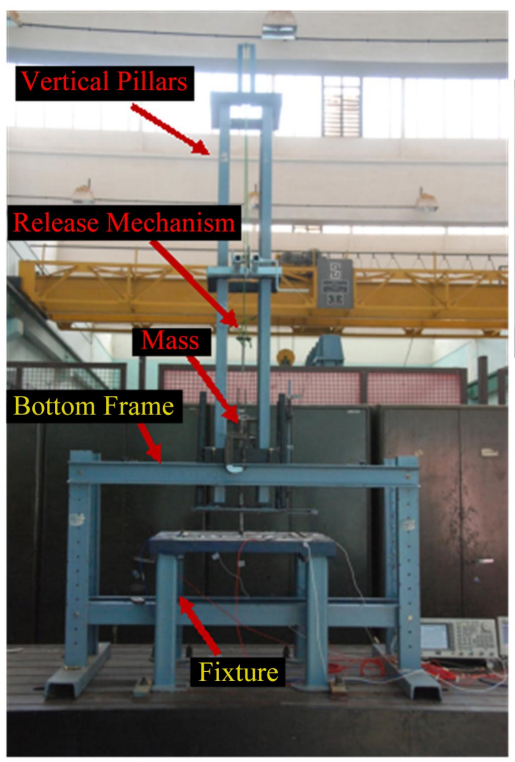

(b)
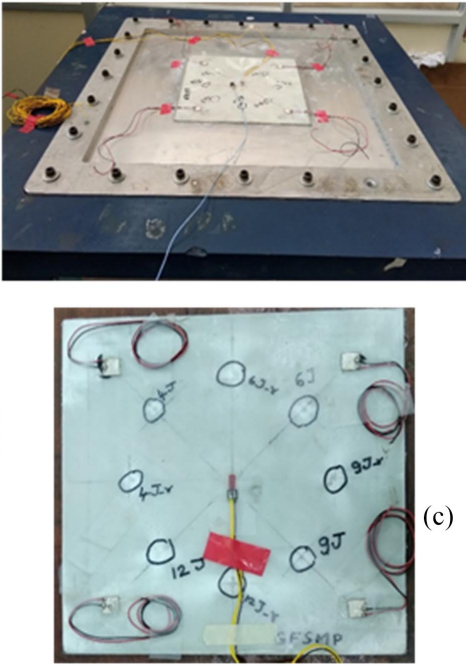

(a)

Figure 3. Low velocity impact testing set-up.

It is to be noted that, the response signals have both low frequency and very high frequency components. Also, the number of samples considered is very high; thus the data acquisition, data handling and data processing have become really a challenging. The idea is to evaluate the transient dynamic behaviour of flexible resin based green composite. In particular, for impact damage monitoring, which resin system is useful to transmit the high frequency lamb waves. As it is known that lamb waves carry the micro level damage information, which can be easily retrieved by signal processing tools. A simple PZT patches-based network is attempted in the present study with four numbers of patches to collect the impact responses. In the next section, the signal collection and processing method is discussed.

\section{Signal Analysis Methodology}

Four PZT patches are bonded at the specified locations on the surface of GFRP and GF-SMP panels. Since PZT patches are strain based transducers, they can be able to capture the vibration signatures due to impact loading, as voltage time signals (Direct Piezoelectric Effect). The NI PXIe data acquisition system, featured with the PXIe 1082 chassis and PXIe 5105 8-channel digitizing oscilloscope, enables the impact data acquisition through trigger event mode at the sampling rate of $60 \mathrm{MHz}$ for each channel. The time duration of each channel i.e. 4 channels in the recorded data is $0.1667 \mathrm{sec}$ with a record length of $10^{7} \mathrm{sam}$ ples and the data are saved in "lvm" file format. Since the memory size of "lvm" file for this particular data is above $1 \mathrm{~GB}$, a file format conversion program is developed in Matlab ${ }^{\star}$ to change the data file into "mat" file format for the ease of data accessing.

It is possible to theoretically estimate the power spectrum of a signal, which is 
sampled at $60 \mathrm{MHz}$ in the band of dc to $30 \mathrm{MHz}$; however, the bandwidth chosen to characterize the material behaviour of the panels in the structural dominant frequencies during the impact is $20-2000 \mathrm{~Hz}$. As the data acquisition is a one-time event, signal conditioning is performed, while post-processing, unlike any standard test-measurement equipment. The noise in the signal is distributed across all frequencies in the FFT spectrum, bringing down the signal-to-noise ratio. And to improve the signal's quality, it demands several higher order digital filters (higher order polynomials - more filter coefficients) due to higher sampling rate, eventually leading to a computationally expensive signal analysis.

Wavelet transformation is an effective way of implementing multi-rate filter banks. Through continuous wavelet transform (CWT), narrow bandpass filtering for multiple frequencies are implemented digitally as an alternate to multiple higher order digital filters. Wavelet coefficients are obtained by CWT as a two-dimensional joint time-frequency distribution variable along with frequencies, adopting a suitable scale factor, scaled to sampling frequency appropriately. To obtain the two-dimensional wavelet coefficients, an inverse continuous wavelet transform (ICWT) of a particular frequency (defined through Narrow Band with the said frequency at its centre) is applied, which results in the time domain signal that is clean and dominant in the given frequency.

However, when CWT of the signal, sampled at $60 \mathrm{MHz}$ is processed, the frequency vector obtained from the transformation has produced a sparse distribution of the chosen band i.e. in the band of $20-2000 \mathrm{~Hz}$. Only a few data points are calculated, which are insufficient to perform the signal analysis. It is observed that this may be due to the limitation of the in-built function, which cannot be customized. In order to achieve an optimum frequency resolution for the needed bandwidth by CWT, resampling of PZT signals through new sampling rate, following Nyquist criteria is performed. After that the individual frequency components are extracted and then the power spectral density is estimated by Welch method [21]. This has resulted in several spectra, based on the number of frequencies, defined in $20-2000 \mathrm{~Hz}$. They are subsequently summed up to obtain the aggregate power spectral density of the conditioned PZT signals.

\section{Mathematics of Signal Processing}

\section{Resampling Process:}

Let us consider the acquired signal as $x_{i}\left(n_{1}\right)$ where $i=1$ to 4 , which represents the number of channels and $n_{1}$ is the sample indices, generated due to sampling rate $F_{s}^{1}=60 \mathrm{MHz}$. The frequency band of interest is 20 to $2000 \mathrm{~Hz}$; therefore, resampling frequency is done with $F_{s}^{2}=10 \mathrm{kHz}$. Accordingly, the new sampling indices are represented as $n$. Now the resampling is performed by combining the effect of interpolation and decimation, through the factors $L$ and $M$, respectively, using the rational factor $L / M$. A low pass filter is used, whose cut-off is minimum $\pi / L$ and $\pi / M$ with a DC gain $L$. The resampling or sampling rate conversion is illustrated in Figure 4(a). 


\section{Signal Conditioning through Multirate Filter Banks.}

The continuous wavelet transformation of $x_{i}(n)$ is given by the following relation $\left[W_{i}, f_{\Omega}\right]=C W T\left\{x_{i}(n), w\left(n^{*}\right)\right\}$, where $W_{i}$ is the complex wavelet coefficients, $f_{\Omega}$ is the frequency, scaled to resampling frequency, and $w\left(n^{*}\right)$ is the wavelet filter with window size $n^{*}$. The continuous inverse wavelet transformation function needs a definite lobe or a narrow band, to feed a particular frequency as input parameter i.e. it requires both $f+\Delta f$ and $-\nabla f$, instead of frequency $(f)$. Hence, the frequency band of interest is represented in two vectors $F_{1}$ and $F_{2}$. These frequency vectors are defined by the following relations; $F_{1}(j)=\left[f_{1}^{c}-f_{l}: f_{\text {step }}: f_{2}^{c}-f_{l}\right]$ and $F_{2}(j)=\left[f_{2}^{c}+f_{l}: f_{\text {step }}: f_{2}^{c}+f_{l}\right]$, where $f_{1}^{c}$ and $f_{2}^{c}$ are the lower and upper cut off frequencies, with $f_{\text {step }}$ is the frequency step, $j$ is the frequency vector index and $f_{l}$ is the $\nabla f$, defining the lobe/narrow band. The inverse transformation is given by the relation: $R_{i}(n, j)=I C W T\left\{W_{i}, f_{\Omega}, F_{1}(j), F_{2}(j)\right.$, mean $\left.\left(x_{i}(n)\right)\right\}$. Through iterative inverse continuous wavelet transformation, four individual two-dimensional variables $R(n, j)$ are extracted, whose rows represent the time domain sample index and the columns define the frequencies in the band of interest. The signal conditioning is illustrated in Figure 4(b).

\section{Power Spectral Estimation:}

The two-dimensional $R_{i}(n, j)$ variables, represent the time domain signal of different frequencies, extracted through multi-band pass filtering by continuous wavelet transform. It is applied for spectral analysis. The Welch method of PSD estimation [21] is given by the function $\left[P_{i}^{x}(j), f^{x}\right]=\operatorname{PWelch}\left\{R_{i}(n, j), F_{s}^{2}\right\}$. The aggregate PSD is accordingly calculated by summing the PSD, resulting from $1^{\text {st }}$ frequency to $f^{\text {th }}$ frequency, using the relation $P_{i}=\sum_{k=1}^{j} P_{x}(k)$. The PSD estimation is illustrated in Figure 4(c).

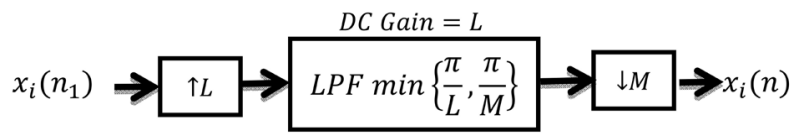

(a)

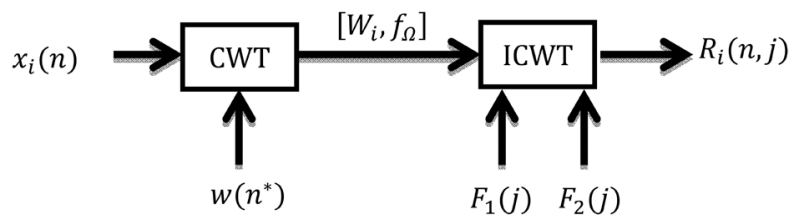

(b)

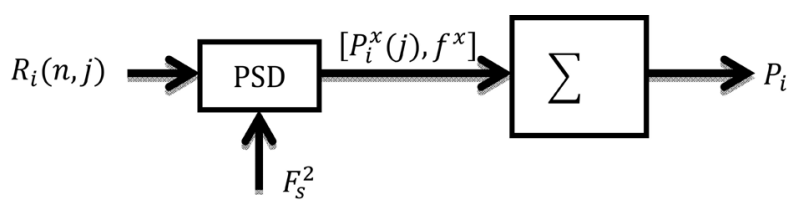

(c)

Figure 4. (a) Block diagram of signal processing; (b) Block diagram of signal conditioning; (c) Block diagram of PSD estimation. 


\section{Results \& Discussion}

The transient responses obtained from low velocity impact tests have been processed using the methodology, described in Section 4. The location of the four PZT sensors is already shown in Figure 3(c). The locations of the impact events can be seen from this figure. The measurements are made for different impact intensities, namely 4J, $6 \mathrm{~J}$ and 12J. The PSD plots are presented in two frequency bands, namely 20 to $2000 \mathrm{~Hz}$ to show the structural frequencies and 10 to 100 $\mathrm{KHz}$, to display the generated lamb waves by impact events. As it is explained earlier that the main objective of this research study is to understand the transient response of green composite, made up of shape memory polymer. Further, it is felt that a comparison with a standard polymer system would provide useful technical merits of this new composite (GF-SMP).

The results are shown in Figures 5-10, in the form of PSD plots. It is evident that, in the structural frequency band $(0-2000 \mathrm{~Hz})$, in general, GF-SMP could better damp-out the energy due to impact (refer to Figure 5, Figure 7 and Figure 9). It is to be noted that SMP has got higher toughness and loss modulus, compared to epoxy [19], and because of that, the panel vibrations are quickly dissipated in the case of GF-SMP. It is further interesting to see that the newly developed DSP based approach is able to produce a very useful frequency domain information about the generated high frequency waves by impact events.

It can also be observed that both GF-SMP and CFRP have generated the lamb waves sufficiently under the impact loading. However, in the low intensity impact event (4J), GF-SMP has developed better response information. As it is known that BVID (Barely Visible Impact Damage) usually occurs due to low intensity impact, mostly introducing matrix cracks or fibre misalignment. Further, it is to be noted that lamb waves have the capability to capture such BVID information.
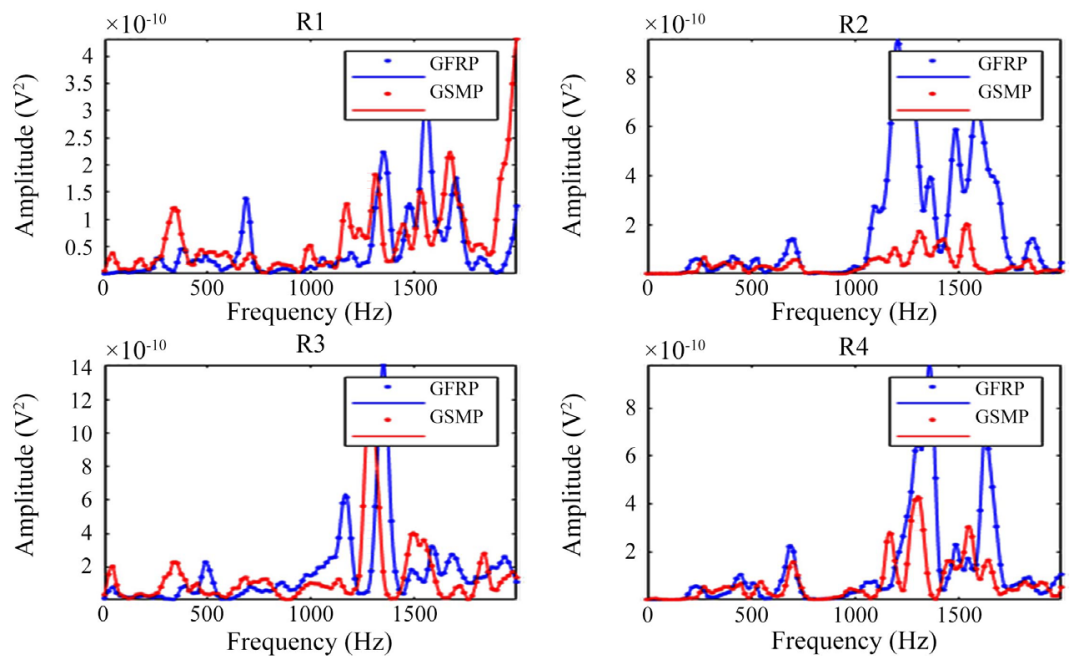

Figure 5. Power spectral density plot for 4 Joule Impact $(20$ - $2000 \mathrm{~Hz}$ Band). 

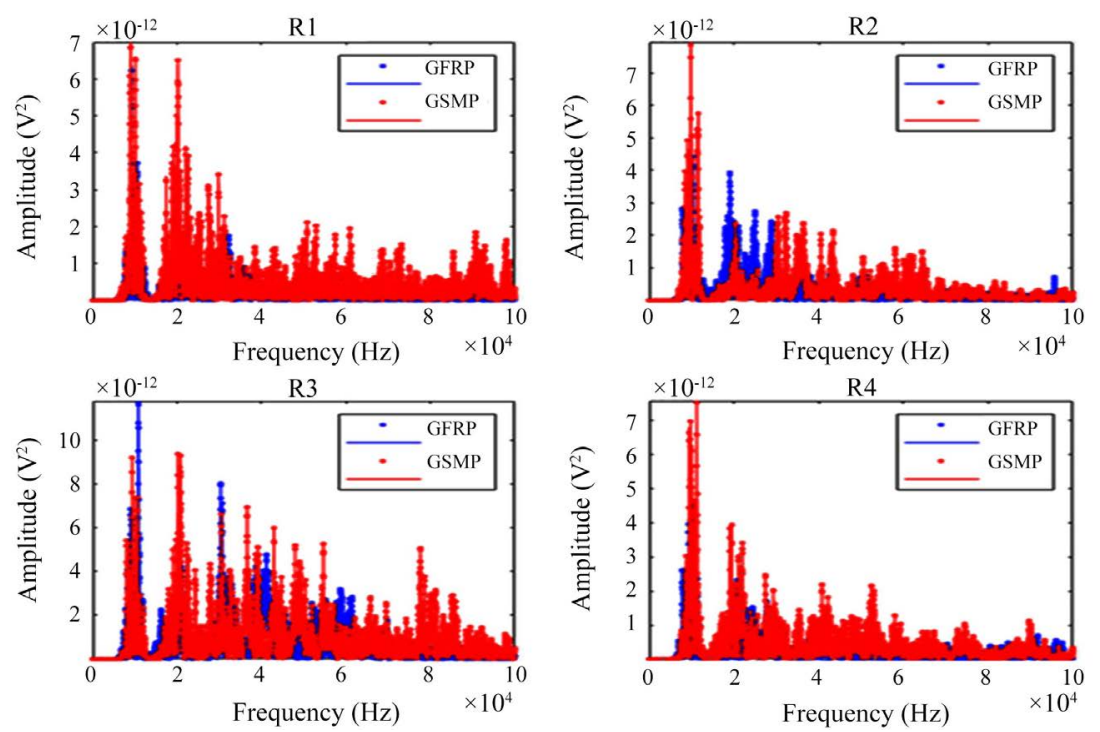

Figure 6. Power spectral density plot for 4 Joule Impact (10 - $100 \mathrm{KHz}$ Band).
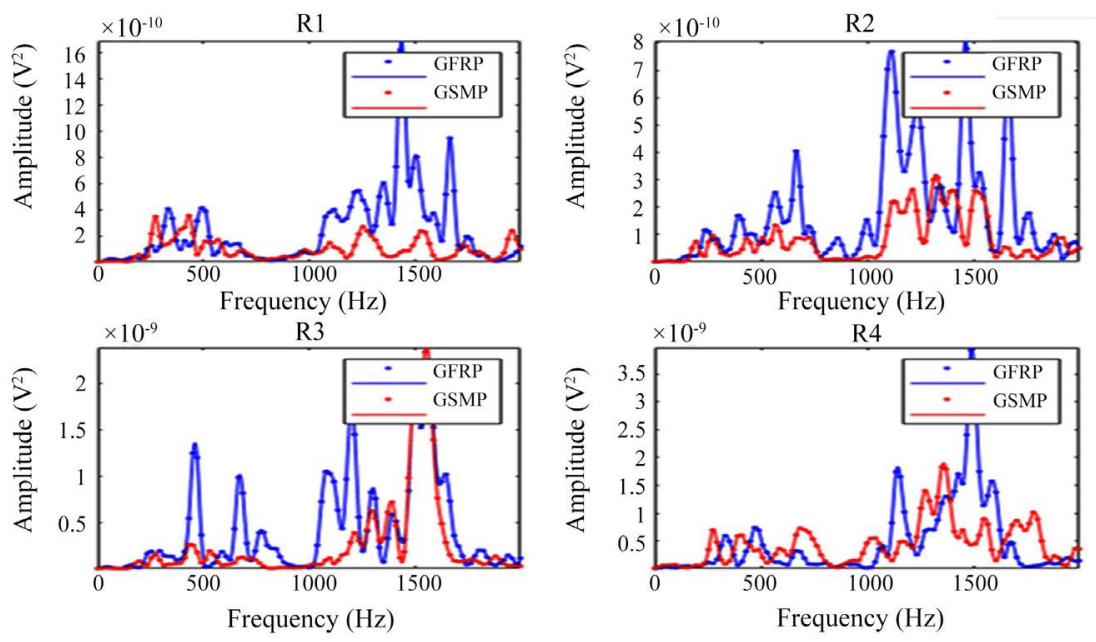

Figure 7. Power spectral density plot for 6 Joule Impact (20 - $2000 \mathrm{~Hz}$ Band).
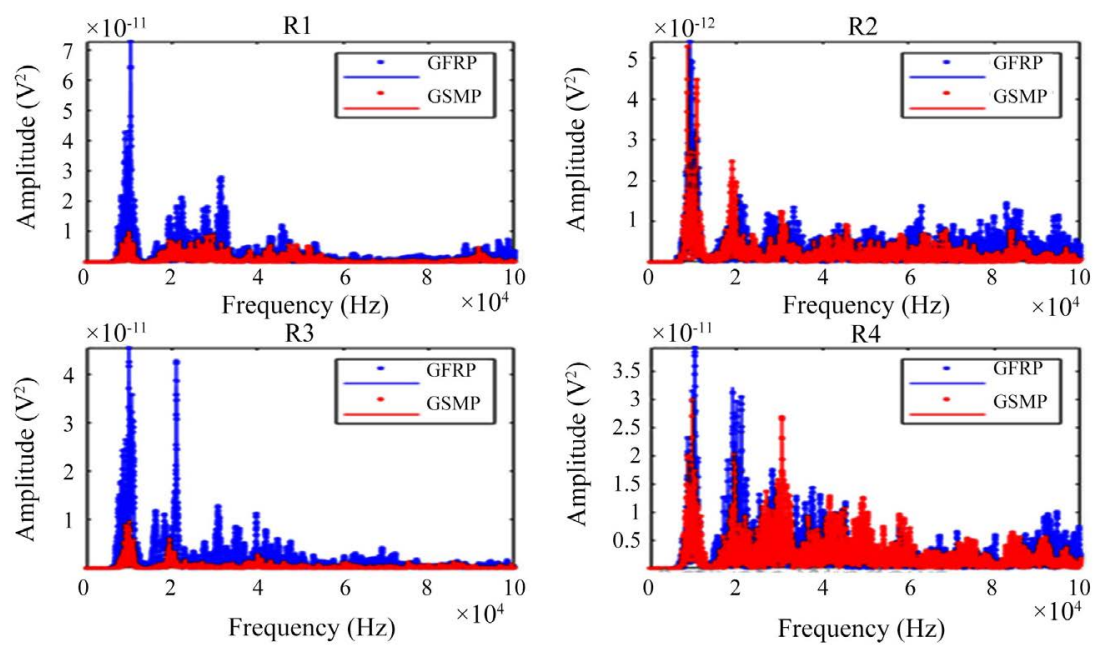

Figure 8. Power spectral density plot for 6 Joule Impact (10 - $100 \mathrm{KHz}$ Band). 

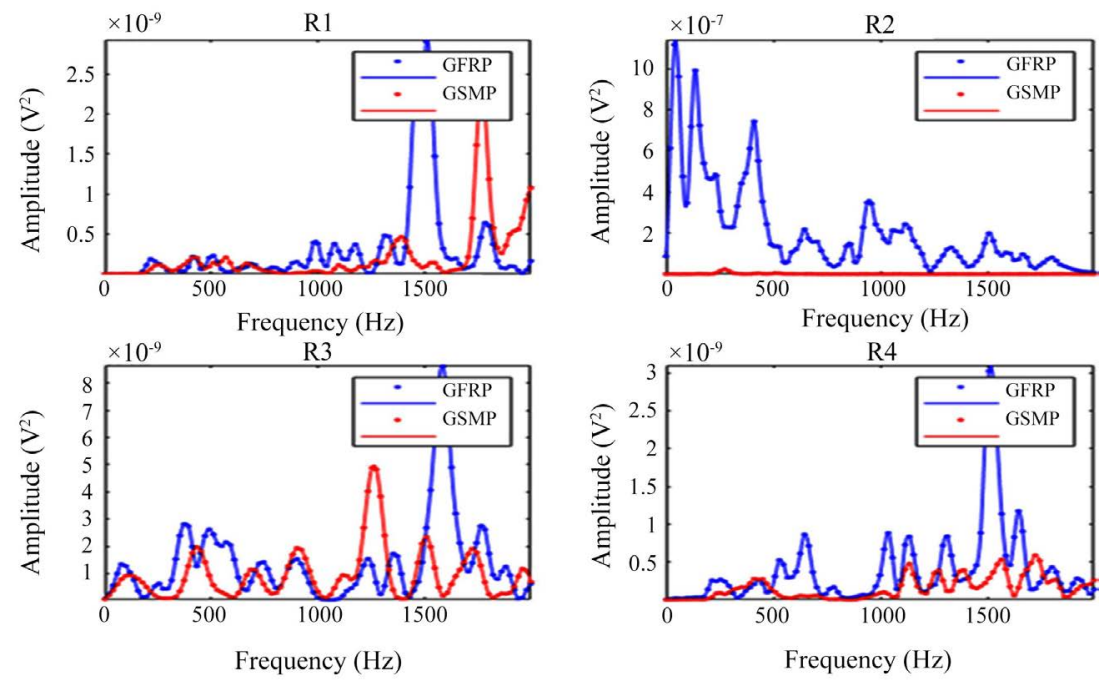

Figure 9. Power spectral density plot for 12 Joule Impact (20 - $2000 \mathrm{~Hz}$ Band).
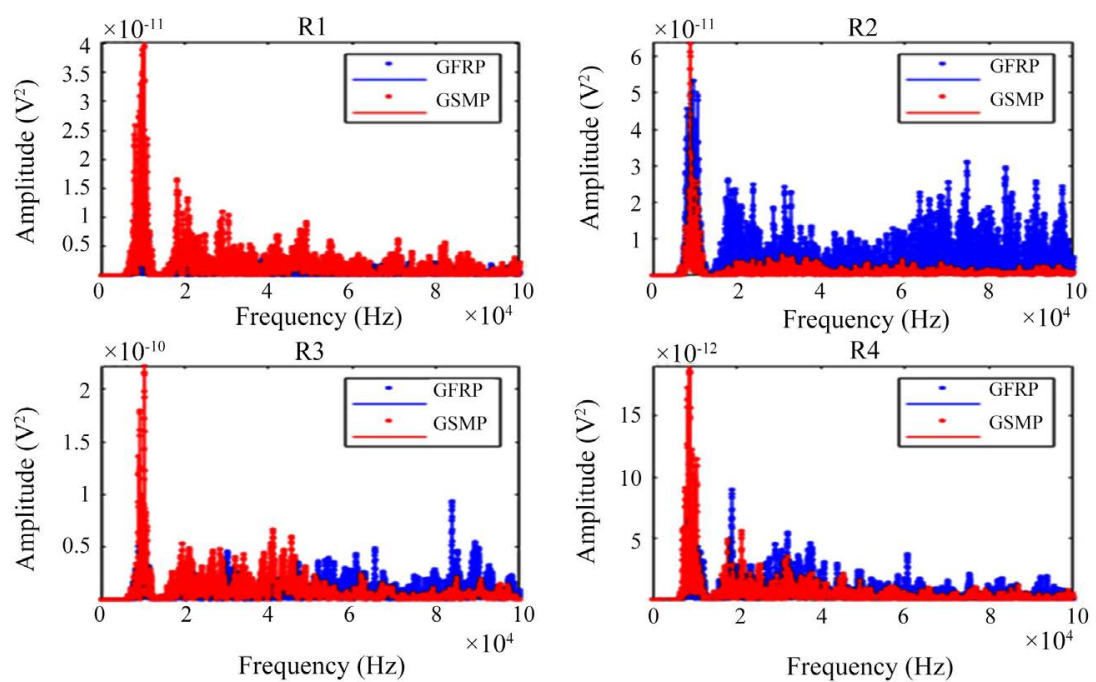

Figure 10. Power spectral density plot for 12 Joule Impact (10 - $100 \mathrm{KHz}$ Band).

Therefore, GF-SMP composites have got appreciable features, both in structural frequency and high frequency lamb wave bandwidths. Most of the urban utility UAV's fly in the altitudes of $<500 \mathrm{~m}$, which are prone to bird strike kind of impact event. The GF-SMP may be used to build such UAV structures with impact event monitoring feature.

\section{Conclusions}

In this research work, the impact behaviours of two laminated composites, namely GFRP with a regular epoxy resin and GF-SMP using an indigenously processed shape memory polymer [19] are presented. Low velocity impact tests were conducted on these composite specimens with different intensities of impact (4J, 6J, 12J). The transient responses were monitored with the help of four PZT patches and later, the obtained responses were processed in two frequency 
bands, i.e., 20 to $2000 \mathrm{~Hz}$ and 10 to $100 \mathrm{KHz}$, in order to study the structural frequencies and high frequency lamb waves.

GF-SMP has shown a better damping nature, compared to GFRP. Also, the high frequency lamb waves due to impact events are clearly separated using the proposed digital signal processing approach in the present study.

It has been demonstrated that, an impact event may be easily monitored by a network of PZT patches online using the developed scheme. Further, it is worth noticing that GF-SMP has shown a potential application to be used as leading edges to resist the impact related loadings in UAV structures, instead of aluminum metal.

\section{Conflicts of Interest}

The authors declare no conflicts of interest regarding the publication of this paper.

\section{References}

[1] Filho, L.M.R.S., Oliveira, P.R., Panzera, T.H. and Scarpa, F. (2019) Impact of Hybrid Composites Founded on Rubber Tyres Particles and Sugarcane Bagasse Fibres. Composites Part B: Engineering, 159, 157-164. https://doi.org/10.1016/j.compositesb.2018.09.054

[2] Richardson, M.O.W. and Wisheart, M.J. (1996) Review of Low-Velocity Impact Properties of Composite Materials. Composites Part A: Applied Science and Manufacturing, 27, 1123 -1131. https://doi.org/10.1016/1359-835X(96)00074-7

[3] Long, S.C., Yao, X.H. and Zhang, X.Q. (2015) Delamination Prediction in Composite Laminates under Low-Velocity Impact. Composite Structures, 132, 290-298. https://doi.org/10.1016/j.compstruct.2015.05.037

[4] Polimeno, U. and Meo, M. (2009) Detecting Barely Visible Impact Damage Detection on Aircraft Composite Structures. Composite Structures, 91, 398-402. https://doi.org/10.1016/j.compstruct.2009.04.014

[5] Xu, S. and Chen, P.H. (2013) Prediction of Low Velocity Impact Damage in Carbon/Epoxy Laminates. Procedia Engineering, 67, 489-496. https://doi.org/10.1016/j.proeng.2013.12.049

[6] Batra, R.C., Gopinath, G. and Zheng, J.Q. (2012) Damage and Failure in Low Energy Impact of Fiber-Reinforced Polymeric Composite Laminates. Composite Structures, 94, 540-547. https://doi.org/10.1016/j.compstruct.2011.08.015

[7] Kadlec, M. and Ruzek, R. (2011) A Comparison of Laser Shearography and C-Scan for Assessing a Glass/Epoxy Laminate Impact Damage. Applied Composite Materials, 19, 393-407. https://doi.org/10.1007/s10443-011-9211-1

[8] Jang, B.W. and Kim, C.G. (2017) Real Time Detection of Low Velocity Impact Induced Delamination Onset in Composite Laminates for Efficient Management of Structural Health. Composites Part B: Engineering, 123, 124-135. https://doi.org/10.1016/j.compositesb.2017.05.019

[9] Chen, Y., Hou, S., Fu, K., et al. (2017) Low Velocity Impact Response of Composite Sandwich Structures: Modelling and Experiment. Composite Structures, 168, 322-334. https://doi.org/10.1016/j.compstruct.2017.02.064

[10] Destic, F. and Bouvet, C. (2016) Impact Damages Detection on Composite Mate- 
rials by THz Imaging. Case Studies in Nondestructive Testing \& Evaluation, 6, 53-62. https://doi.org/10.1016/j.csndt.2016.09.003

[11] Anton, S.R., Butland, A., Carrion, M., Buechler, M. and Park, G. (2007) Instantaneous Structural Damage Identification Using Piezoelectric-Based Lamb Wave Propagation. IMAC XXV, SEM.

[12] Inman, D.J., Farrar, C.R., Lopes Jr., V. and Steffen Jr., V. (2005) Damage Prognosis for Aerospace, Civil and Mechanical Systems. John Wiley and Sons, Ltd., West Sussex. https://doi.org/10.1002/0470869097

[13] Diamanti, K., Hodgkinson, J.M. and Soutis, C. (2004) Detection of Low Velocity Damage in Composite Plates Using Lamb Waves. Structural Health Monitoring, 3, 33-41. https://doi.org/10.1177/1475921704041869

[14] Raghavan, A. and Carlos Cesnik, E.S. (2007) Review of Guided-Wave Structural Health Monitoring. The Shock and Vibration Digest, 39, 91-114.

[15] Su, Z.Q., Ye, L. and Lu, Y. (2006) Guided Lamb Waves for Identification of Damage in Composite Structures: A Review. Journal of Sound and Vibration, 295, 753-780. https://doi.org/10.1016/j.jsv.2006.01.020

[16] Lu, S.W., et al., (2019) Real-Time Monitoring of Low-Velocity Impact Damage for Composite Structures with Omnidirection Carbon Nanotubes' Buckypaper Sensors. Structural Health Monitoring, 18, 454-465. https://doi.org/10.1177/1475921718757937

[17] Ratna, D. and Karger-Kocsis, J. (2007) Recent Advances in Shape Memory Polymers and Composites: A Review. Journal of Materials Science, 43, 254-269. https://doi.org/10.1007/s10853-007-2176-7

[18] Liu, C., Qin, H. and Mather, P.T. (2007) Review of Progress in Shape-Memory Polymers. Journal of Materials Chemistry, 17, 1543-1558. https://doi.org/10.1039/b615954k

[19] Antony, G.J.M., Jarali, C.S., Aruna, S.T. and Raja, S. (2017) Tailored Poly(ethylene) Glycol Dimethacrylate Shape Memory Polymer for Orthopaedic Applications. Journal of the Mechanical Behavior of Biomedical Materials, 65, 857-865. https://doi.org/10.1016/j.jmbbm.2016.10.011

[20] Safranski, D.L. and Griffis, J.C. (2017) Chapter Six-Applications of Shape Memory Polymers. In: Shape-Memory Polymer Device Design, William Andrew, Norwich, NY, 189-222. https://doi.org/10.1016/B978-0-323-37797-3.00006-3

[21] Proakis, J.G. and Manolakis, D.G. (1996) Digital Signal Processing: Principles, Algorithms and Applications. 3rd Edition, Prentice-Hall, Upper Saddle River, 910-913. 